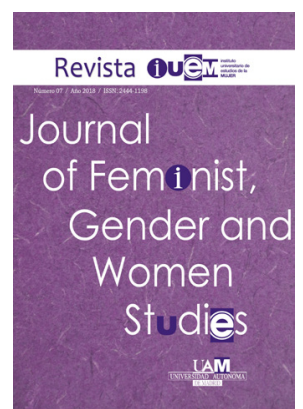

\section{La intimidad es política. Una mirada a los derechos sexuales y reproductivos de las mujeres con diversidad funcional}

\author{
Intimacy is political. Sexual and Reproductive Rights of women with disabilities
}

\author{
Helena Pérez-de la Merced ${ }^{1, @ ~}$
}

${ }^{1}$ Departamento de Psicología Evolutiva y de la Educación. Universidad Autónoma de Madrid. España.

$@$ Autor/a de correspondencia: perezdelamerced@gmail.com

\title{
Resumen
}

Este artículo constituye una reflexión desde una perspectiva feminista y teniendo en cuenta el enfoque interseccional de los principales puntos teóricos del diálogo entre dos construcciones sociales como son el género y la diversidad funcional centrándonos en el aspecto específico de la sexualidad y la reproducción de las mujeres con diversidad funcional.

A partir de estos dos aspectos, se destaca la importancia de generizar la diversidad funcional así como de que el feminismo atienda a las necesidades de las diferentes mujeres, en este caso, de las mujeres con diversidad funcional. Teniendo esta multiplicidad de circunstancias en cuenta, la mirada interseccional emerge como herramienta para dar respuesta a la variedad de demandas en las áreas de los derechos sexuales y de los derechos reproductivos.

Se trata, por tanto, de una análisis de la cuestión sobre el mundo del feminismo y de la diversidad funcional profundizando en sus semejanzas y sus diferencias así como apuntando hacia los futuros retos que nos plantea esta conversación género/diversidad funcional.

Palabras claves: Género, diversidad funcional, sexualidad, maternidad.

\begin{abstract}
This article constitutes a reflection of the principal theoretical key issues as a result of the dialogue between two social constructions: gender and disability from a feminist and intersectional point of view focusing on the sexuality and reproduction of disabled women.

From these two aspects (sexuality and reproduction) gendering disability is a very important point to satisfy the differenciated demands of women, in this case: women with disabilities. Thus, intersectional point of view comes out as a tool to give a response to the variety of demands in the area of sexual and reproductive rights.

Therefore, it is an analysis of disability and feminism issues carrying out more in-depth about their similarities and differences as well as their upcoming challenges.
\end{abstract}

Keywords: Gender, disability, sexuality, motherhood. 


\section{INTRODUCCIÓN}

En 2005 (y durante un año y medio) se erigió en pleno centro de Londres - en Trafalgar Square - la estatua de Alison Lapper realizada por Marc Quinn en la que se observa a una mujer desnuda, embarazada y con diversidad física. Con esta obra el autor pretendía "hacer reaccionar al público" y que "la escultura calara en la gente y suscitara emociones" (EFE, 2005). Echando la vista atrás lo cierto es que esta efigie generó un gran revuelo inicial; algo que no es de extrañar puesto que, como señala Lapper, "aborda todos los prejuicios y tabúes existentes sobre sexualidad, discapacidad, feminidad y embarazo" (EFE, 2005).

Diez años después, en 2015, se estrenó en nuestro país Yes We Fuck, documental que visibiliza la sexualidad de las personas con diversidad funcional. En él se muestra a mujeres y hombres con diversidad funcional como protagonistas - sujetos deseables y deseantes- en entornos eróticos.

Ambas imágenes ponen frente al espejo de la sociedad los estereotipos que planean sobre el imaginario colectivo en relación con los derechos sexuales y reproductivos (DDSSRR) de las personas con diversidad funcional. Si se hace una revisión, es difícil localizar entre las miles de imágenes con las que nos cruzamos a diario el cuerpo con diversidad funcional como sujeto que profiere cuidados o desempeña un rol erótico siendo objeto o sujeto de deseo. Como explica Bonnie con elocuencia (2004), rara vez las personas con discapacidad son retratadas como personas sexualmente activas o como madres y padres. Y es que, ¿cuántas veces hemos visto a personas con discapacidad desfilar por las pasarelas de Londres, Milán o París o aparecer en las páginas de la Cosmo o la Vogue? (Bonnie, 2004: 125). El sexo, por tanto, es representado como un privilegio del hombre blanco, heterosexual, joven, de clase media y sin diversidad funcional. Las representaciones sexuales de personas que son más mayores (de lo que "deberían ser"), más gordxs, con la piel más oscura, gays o con discapacidad han estado ausentes en los medios convencionales (Tepper, 2000: 285). Lo mismo ocurre con el ámbito de la reproducción. Basta realizar una búsqueda en la web de la palabra madre para adivinar quién ostenta el privilegio de ser sujeto reproductivo: mujer de clase media, occidental, sin diversidad funcional, de una determinada edad...Y es que las imágenes de madres o padres con discapacidad son raramente usadas y, cuando lo hacen, ofrecen mensajes mezclados (Wates, 2004).

La vida de las personas con diversidad funcional es más que empleo, atención médica o educación. Pero, ¿por qué las organizaciones no empiezan a discutir y debatir esto? (Bonnie, 2004). La realidad es que gran parte de los planes de intervención olvidan el área sexo-afectiva y reproductiva poniendo énfasis en aspectos académicos y laborales enfocados a la vida productiva pero escasean los programas dirigidos al terreno personal (no productivo) que incluirían necesariamente sus prácticas sexuales y afectivas (Cruz, 2004). Las personas con diversidad funcional a menudo no pueden obtener información básica sobre su salud sexual y reproductiva desconociendo aspectos básicos de ellos y ellas mismas, sus cuerpos y su derecho a definir qué quieren y qué no quieren (OMS, 2009). Privar a estas personas de educación sexual es exponerlas a una mayor vulnerabilidad
(Ministerio de Sanidad, Política Social e Igualdad, 2011). El hecho de no pensar en esta área sexo-afectiva hace que incluso las personas con diversidad funcional heterosexuales lleven a cabo este proceso de "salir del armario" cuando empiezan a afirmar o reivindicar su sexualidad (Bonnie, 2004: 127). Imaginemos pues la doble invisibilidad de los deseos de las personas con diversidad funcional que se escapan de la heteronorma. Fruto de que estas áreas apenas hayan sido transitadas, la lucha por los derechos sexuales y reproductivos emerge como elemento central del activismo de determinados sectores dentro del movimiento por los derechos de las personas con diversidad funcional.

En los últimos años, por su parte, la comunidad internacional ha puesto su atención en la no violación de estos derechos sexuales y reproductivos a través de diferentes convenios y tratados. No obstante, este reconocimiento de iure no va de la mano de su cumplimiento de facto a pesar de que la Convención sobre los Derechos de las Personas con Discapacidad (CDPD) explicita en su artículo 23 que "los Estados Partes tomarán (las) medidas efectivas y pertinentes para poner fin a la discriminación contra las personas con discapacidad en todas las cuestiones relacionadas con el matrimonio, la familia, la paternidad y las relaciones personales, y lograr que las personas con discapacidad estén en igualdad de condiciones con las demás" (Convención sobre los Derechos de las Personas con Discapacidad, 2006).

En las próximas líneas se pone el foco en alguno de los aspectos clave en este diálogo entre dos construcciones sociales: la del género y la de la discapacidad en el ámbito específico de los derechos sexuales y reproductivos.

En el primer apartado, a partir de la expuesta necesidad de atención hacia la materialización de los DDSSRR de las mujeres con diversidad funcional, se destaca la importancia de introducir la perspectiva de género a la hora de analizar la realidad de las personas con diversidad funcional. Con esta mirada de género se consigue desvelar los prejuicios sufridos por las mujeres con diversidad funcional con respecto a los ámbitos que tienen que ver con su sexualidad y su reproducción.

En relación con el segundo apartado y con esta perspectiva de género en mente, se detallan aspectos clave de la feminidad hegemónica que funcionan de manera diferente en mujeres con diversidad funcional. Estas diversas circunstancias, por tanto, proponen diferentes demandas al movimiento feminista mainstrean que lo dirigen hacia el camino de la consecución de un feminismo de todas para todas.

El enfoque interseccionalidad puede resultar un instrumento útil desde un punto de vista tanto teórico como político para identificar y satisfacer esta amalgama de demandas de las mujeres. Este tercer apartado, por tanto, se dedica a prestar atención a la interseccionalidad y a realizar un breve repaso de la misma en lo que tiene que ver los DDSSRR de las mujeres con diversidad funcional en el ámbito español.

Para finalizar, el último apartado concluye poniendo énfasis en la necesidad de atender a la diversidad puesto que todas estamos conformados por una mezcolanza de circunstancias, condiciones y particularidades. Es decir, la diversidad es intrínseca a la condición humana. 


\section{GENDERING DISABILITY}

"En el pasado, tanto las feministas no discapacitadas como los hombres que dominaban el movimiento de las personas discapacitadas han sido ajenos a las preocupaciones de las mujeres discapacitadas. Nuestros encuentros con ambos grupos nos han hecho sentirnos indefensas porque $o$ bien nos han tratado como invisibles o han definido nuestras experiencias por nosotras" (Morris, 1997: 17).

"Los discapacitados piden a Gallardón que elimine la esterilización forzosa" (Publico, 2012). Bajo este titular de una noticia de 2012 aparece la imagen de tres líderes (hombres) de asociaciones de personas con discapacidad pidiendo la derogación del artículo 156 del Código Penal para eliminar la esterilización forzosa de las personas con discapacidad. Para empezar, se debería hablar de personas con discapacidad/ diversidad funcional y no de "discapacitados". Para continuar - yendo hacia delante en el análisis y colocándonos las gafas moradas- vemos, en primer lugar, que son tres hombres quienes ostentan los cargos de responsabilidad en estas asociaciones. En segundo lugar, cabe preguntarse sobre quién se realiza mayoritariamente esta práctica. Pues bien, desagregando las cifras por sexo, según un reciente estudio de la Fundación CERMI Mujeres $(2017,6)$ la esterilización forzosa se ha practicado en especial sobre niñas y mujeres con discapacidad. En este sentido, la CDPD ya advertía que "las mujeres y las niñas con discapacidad suelen estar expuestas a un riesgo mayor, dentro y fuera del hogar, de violencia, lesiones o abuso, abandono o trato negligente, malos tratos o explotación" (Convención sobre los Derechos de las Personas con Discapacidad, 2006).

Como vemos, el presunto velo de neutralidad de género no escapa a la diversidad funcional $y$, por tanto, el género también opera en las diferentes representaciones de hombres y mujeres con diversidad funcional.

La estrecha horma de la feminidad y la masculinidad normativa es un molde al que se torna complicado adecuarse. Desde el momento en que nacemos (o mejor dicho, desde el instante en que se determina y se nombra a cuál de las dos casillas en las que se encuadra el sexo pertenecemos) se generan unas expectativas con respecto a nuestra existencia $y$, por ende, ocupamos uno u otro espacio en el mundo en función de dicha asignación sexual otorgada por la medicina. De acuerdo con el sistema sexo/género enunciado por Gayle Rubin, podríamos decir que se establece una especie de línea de continuidad entre el sexo, el género y la orientación sexual que es la causa de opresión de las mujeres y de las minorías sexuales (Rubin, 1986).

Este sistema heteronormativo encuentra en el dimorfismo sexual (varón/mujer) una especie de esencia que se traduce en destino. Vemos pues cómo este aparato se articula en torno a dos polos reconociendo tan solo dos casillas en la que las personas pueden encuadrarse: mujer o varón. De esta manera, a cada uno y a cada una se le presupone una serie de comportamientos, actitudes, gustos y experiencias diferenciados y, como si de una obra de teatro se tratase, - rescatando el concepto de performance del que nos hablaba Butler - cada uno de los personajes (hombres y mujeres) de esta obra de teatro del género adquiere diferentes elementos del disfraz que le corresponde: gestos, palabras, atuendo, comportamientos...
Dentro de estos estereotipos de género y de las conductas asociadas a cada uno de los sexos, este binarismo ha construido dicotomías resultado de la contraposición hombre/mujer tales como razón/naturaleza, independencia/ dependencia o público/privado, etc. A la luz de este modelo, razón, independencia y lo público han sido dotados de un mayor valor social. Dentro del crisol de actitudes, comportamientos y parcelas de la vida, esta feminidad hegemónica posee aspectos clave como: 1) la belleza; 2) el cuidado de otras personas; y 3) la maternidad.

Cabe mencionar que este sistema binario empapa todos y cada uno de los aspectos que rodean la existencia de las personas en sus diferentes ciclos vitales. Además, ejerce una serie de violencias más o menos evidentes, más o menos intensas a todos aquellos disidentes (voluntarixs o no voluntarixs) que transgreden este aparato normativo o a todo aquel que se salga de la norma imperante y podríamos afirmar que no cumplir con la norma en cualquiera de sus escalones (sexo/género/orientación sexual) supone ser rara/o, antinatural, innombrable, pecaminosa...

Las personas con diversidad funcional transgreden el aparato normativo del sistema sexo/género y sus cuerpos abyectos forman parte de lo otro, de aquello que se aleja de la norma. En el caso de las mujeres, la alianza patriarcado capacitismo hace de las suyas a través representaciones sociales imbuidas de ciertos prejuicios hacia su sexualidad y reproducción.

Para comenzar, se hace especialmente complicado encajar en el concepto de feminidad hegemónica que exalta el valor de la belleza. En una sociedad capacitista que ensalza la perfección de los cuerpos (capacitados) se hace difícil responder al mandato de género y adecuarse a un canon de belleza que define el atractivo femenino del que se supone, como objetos susceptibles de ser deseados que son los cuerpos femeninos, debe caracterizar a las mujeres.

En cuanto a la maternidad, esta se presenta en este sistema heteronormativo como la "profesión femenina por excelencia" impregnando todo un universo/orden simbólico en el que aparece como eje central que define la feminidad. Como vemos, del hecho biológico de poder tener hijos se han extraído muchas de las características que establecen la definición de feminidad (Rich, 1996). Esta feminización de los cuidados se extrapola incluso al ámbito profesional y las mujeres acaban siendo legión en profesiones como enfermera o maestra o tejiendo cadenas globales de cuidados que nos hacen ver cómo la división sexual del trabajo impregna nuestra organización social. La capacidad del cuidado debería ser terreno universal de todas las personas y no ámbito restringido a las mujeres.

Por otro lado, la identidad de madre y la de cuidadora supondrían caras de una misma moneda: la identidad de mujer (visión mujer/madre/cuidadora). La sociedad espera de las mujeres que concurran en la feminidad y en todos los valores asociados a ella. Espera, en definitiva, que concurran en la maternidad. Sin embargo, no espera que las mujeres con diversidad funcional acaben siendo madres.

Por tanto, estos estereotipos funcionan de forma diferente en las mujeres con diversidad funcional. Como ya se ha mencionado, en nuestro sistema patriarcal la maternidad aparece de forma más o menos velada como piedra angular de la feminidad teniendo el modelo de mujer cuidadora como 
parte clave del mismo. A través de la siguiente experiencia ilustramos cómo la vivencia varía en estas mujeres con diversidad funcional:

"Nadie me dijo nunca que podía tener hijos. Casi todas las mujeres que yo conocía eran madres, pero ninguna de estas madres era ciega. Yo solo conocía a dos mujeres adultas ciegas, y ninguna tenía hijos. En mi joven mente esta muestra se trasladaba a todo el mundo: las mujeres videntes eran madres; las mujeres ciegas, no"(Kent, 2002:81).

Entre los mitos que circulan en el imaginario colectivo en torno a la maternidad de las mujeres con diversidad funcional (López, 2008; Kalender, 2010; O’Toole, 2002) podemos citar: 1) el mito de la asexualidad (mujer con diversidad funcional no es ni objeto ni sujeto de deseo); 2) la creencia de que "la diversidad funcional se encuentra asociada a la dependencia y el aislamiento, es difícil para la gente imaginar a una persona con diversidad funcional en el centro de la familia como cuidadora o cuidador" (Morris, 1997); 3) el mito de la discapacidad que se hereda (de madres con diversidad funcional nacerán hijos e hijas con diversidad funcional) y 4) la creencia de que los hijos e hijas de progenitores con diversidad funcional sufren consecuencias psicoemocionales (especialmente si se trata de la madre).

Estos estereotipos son eso: una visión simplificada y sin matices de la realidad. Empero, como sabemos, una cosa son las ideas en un plano abstracto y otra, diferente, las experiencias de la vida cotidiana. Huyendo del relato de la "doble desventaja" que victimiza a las mujeres con diversidad funcional y aportando una visión realista y empírica, según diferentes estudios que dan voz a estas mujeres, estos estereotipos chocan con la realidad. Uno de los principales hallazgos del estudio de Cruz (2004) fue que pese a que socialmente se asignan normas y valores diferenciados a las mujeres con discapacidad y a que existe desigualdad en el acceso a recursos sociales y afectivos, la discapacidad no constituye un factor que imposibilite las prácticas eróticoafectivas. En la misma línea, en el estudio de López (2008) sobre relaciones de pareja y maternidad, las mujeres entrevistadas manifiestan el peso de los prejuicios pero a la vez logran desafiarlos y romper estas ideas preconcebidas. Con respecto a la maternidad, una de las ideas principales es la normalidad de su experiencia a pesar de la discapacidad (Walsh-Gallagher, Sinclair, y Mc Conkey, 2012) y que, pese a las barreras, las mujeres con diversidad funcional abren caminos creativos para hacer ver que cumplen con ese ideal de maternidad que a menudo la sociedad les niega (Malacrida, 2009).

En suma, entender el funcionamiento de estas dinámicas de género dentro del mundo de la diversidad funcional resulta crucial en cualquier proyecto que ansíe la justicia social. Una de las aportaciones de la Fundación Cermi Mujeres al último borrador de la Observación General del Comité sobre los Derechos de las Personas con Discapacidad en materia de implementación y monitoreo de la CRPD señala la necesidad de corregir "los desequilibrios de género en la actividad diaria de las organizaciones que trabajan los asuntos de la discapacidad" puesto que la existencia de estos "fomenta la discriminación directa o indirecta por razones de sexo en tanto en cuanto a la participación económica, política y social de las mujeres y niñas con discapacidad" (Fundación Cermi Mujeres, 2018: 3). "Gendering disability"
(Shakespeare, 1999) o generizar la discapacidad/diversidad funcional no es más ni menos que poner la lupa morada sobre la construcción social de la discapacidad para dar respuesta a las necesidades específicas de estas mujeres y niñas, en este caso, en lo que respecta a sus derechos sexuales y reproductivos (DDSSRR).

\section{DISABLING FEMINISM}

En el ámbito de los DDSSRR, la Organización Mundial de la Salud advierte que la salud sexual y reproductiva "está estrechamente relacionada con diversos factores socioculturales, las funciones características de uno u otro sexo y el respeto y la protección de los derechos humanos, en especial-aunque no exclusivamente- los relacionados con la sexualidad y las relaciones interpersonales" (Organización Mundial de la Salud, 2003: 4). Teniendo en cuenta lo anterior, resulta evidente pensar que las demandas de las mujeres en esta área no son homogéneas. Por ejemplo, durante el gobierno de Gallardón, el Tren de la Libertad representó un triunfo impulsado por el feminismo mainstream pidiendo al ministro la retirada de las modificaciones sobre la Ley del Aborto por considerarlas una involución de los derechos sexuales y reproductivos de las mujeres. En el anterior apartado hemos visto, por otro lado, cómo una de las demandas principales de las mujeres con diversidad funcional es la de poner fin a las esterilizaciones forzosas. El aborto es otro tema con diferentes perspectivas para el feminismo mainstream y para el movimiento de las personas con diversidad funcional. Estas últimas critican el aborto selectivo y el examen prenatal como prácticas eugenésicas (Shakespeare, 1998 en Sheldon, 2004) y las primeras, sin embargo, lo han visto como otro medio más a través del cual ganar control sobre su reproducción (Bailey, 1996 en Sheldon, 2004). ¿Cómo entender la lucha de las mujeres con diversidad funcional por sus derechos sexuales y reproductivos? ¿Cómo entender que mientras gran parte del feminismo está luchando por el derecho al aborto y la desfeminización del ámbito del cuidado, las mujeres con diversidad funcional luchen por su derecho a ser madres y a ejercer el cuidado? La explicación pasa por poner el foco en el hecho de que el cuerpo femenino discapacitado ha sido sometido a un proceso de desexualizacion y desfeminizacion. De ahí que la cuestión de feminidad y de la maternidad conforme una lucha estratégica que nada tiene que ver con la lucha contra la maternidad obligatoria o el aborto de los movimientos feministas. Habrá que analizar pues si se trata pues de una cuestión de resistencia y crítica a la norma o identificación con el modelo tradicional de mujer (Preciado, 2011).

Por tanto, en cuanto a DDSSRR se trata, la agenda del movimiento mainstream y la de las mujeres con diversidad funcional difiere en determinados puntos. La realidad es que el feminismo en muchas ocasiones no ha tenido en cuenta las experiencias de las mujeres con diversidad funcional. Recordando el movimiento, en los años 80, el sujeto feminista ya no atiende a la lógica "Nosotras" contra "Ellos" sino que es sustituido por el debate unidad/diversidad del cual saldrán dos corrientes: una antiesencialista (Butler) y otra multiculturalista (Casado, 1998: 77). Otras mujeres chicanas, negras, lesbianas, transexuales, prostitutas, pobres, 
mujeres inmigrantes ilegales o la rebelión que Despentes denominó "el proletariado del feminismo" alzan sus voces (Trujillo, 2009:162). Se deja claro que las diferencias estaban ahí y que no podía negárselas: diferentes clases sociales, etnias, orientaciones sexuales, creencias religiosas, edades o ideologías políticas irrumpen con fuerza en los grupos de mujeres inaugurando así una fisura importante en ese sujeto homogéneo (Casado, 1998), esto es, mujer blanca, de clase media y heterosexual. Así como el feminismo vino a cuestionar la presunta universalidad del sujeto hombre, el feminismo después hará lo mismo dentro de él mismo con voces que denunciarán la presunta universalidad de la "mujer".

Parafraseando a Shakespeare (1999) se hace asimismo necesario diversitar el feminismo (disabling feminism). Tanto el género como la diversidad funcional son construcciones sociales que parten de la idea de que son las actitudes y las instituciones de las personas sin diversidad funcional y no los atributos físicos lo que convierte las características en hándicaps (Ash y Fine, 1992). Desde esta visión, diversitar la feminidad y la masculinidad representa una oportunidad para tumbar los férreos mandatos de género en los que ninguna de nosotras encajamos. Además, la diversidad funcional guarda relación con otras áreas de investigación en las que el feminismo está trabajando (Morris, 2008).

Como hemos visto, las luchas feministas para mantener la decisión de la reproducción y la de las personas con diversidad funcional para fijar sus derechos de vida, apoyo y oportunidades se han enfrentado, pero esta diferencia en las demandas de los derechos reproductivos supone un diálogo constante que enriquece al feminismo (Ash y Fine, 1992). El feminismo es heterogéneo por definición y dentro de este marco han surgido desacuerdos, disensiones y desavenencias. El movimiento feminista ha avanzado en el camino de su teoría y de su práctica a través de las distintas piedras que se le iban cruzando y que eran lanzadas desde su misma senda. Por tanto, de lo que no cabe duda es que el feminismo es un movimiento vivo, enérgico, vibrante y en constante evolución. Ha sido objeto de contestaciones y críticas que, lejos de mermarlo, le han servido como acicate y lo han empujado hacia una reflexión crítica, siendo a través de estos disensos conceptuales cómo el feminismo ha ido dibujando su línea vital.

En definitiva, el reto pasa por crear un feminismo inclusivo y no un feminismo compartimentado entendiendo que la lucha no es estrictamente sobre el patriarcado o sobre el sistema capitalista sino que la complejidad del mundo actual entreteje alianzas en un continuo de poder entre diferentes sistemas de opresión como el capitalismo, el heteropatriarcado, el capacitismo, el racismo...El feminismo debe entender que estas amenazas requieren de un contraataque que incluya una lucha sinfónica.

\section{PRÓXIMA PARADA: INTERSECCIONALIDAD}

\section{Interseccionalidad estructural}

Cuando hablamos de grupos humanos tendemos a hacer un totum revolutum y solemos referirnos, en el caso que nos ocupa, a: "personas con diversidad funcional", "mujeres", "mujeres con discapacidad", etc. Qué duda cabe, la homogenización resulta útil para crear etiquetas que logren organizar el mundo, pero la realidad se corresponde más bien con una amalgama de experiencias. Esto hace que dentro de cada grupo reine la diversidad frente a la concepción de homogeneidad del conjunto. En el caso de la investigación sobre mujeres con discapacidad, la mayoría opera con un discurso esencializado asumiendo que las mujeres con discapacidad son una categoría monolítica (Wendell, 1996; Ash, Rousso y Jefferies, 2001 en Malacrida 2009: 744). No obstante, estas tienen una variedad de vivencias dependiendo de factores sociales tales como el estatus socio-económico, la ubicación geográfica o su acceso al capital cultural, social y simbólico (Malacrida, 2009). Como apunta Arnau (2005), se debe prestar atención a la inclusión de las "distintas voces de las mujeres con diversidad funcional para no reducir a la mujer con diversidad funcional a mujer con diversidad funcional física, blanca, occidental, heterosexual, de clase media, de mediana edad y de tradición judeo-cristiana" (Arnau, 2005: 23).

En los apartados anteriores hemos puesto el foco en dos construcciones, la de género y la de la diversidad funcional que dialogan y generan determinadas dinámicas, pero otros ejes de exclusión/inclusión no escapan al análisis. Como hemos mencionado se trata de huir del relato de la doble discriminación y prestar atención a quién tiene mayor o menor acceso a recursos sociales y afectivos, como los denomina Cruz (2004). En este punto, aparece la interseccionalidad estructural - acuñada por Crenshaw (1994)- siendo aquella que nos indica la desigualdad interseccional, es decir, las desigualdades que sufre una persona no se manifiestan de forma aislada sino que la interacción de estas determina la situación de una persona en un determinado momento. La novedad e interés del planteamiento de la interseccionalidad radica en que pretende ir al fondo y quitar el velo de la presunta homogeneidad de los grupos sociales a través de las múltiples identidades (a veces ventajosas y a veces no) que forman a los sujetos.

A modo de ejemplo, según el INE (2008: 29), de 20 a 59 años la probabilidad de que las mujeres con discapacidades estén casadas es un $15 \%$ inferior a la de las mujeres sin discapacidades. En el grupo de 60 y más años, la probabilidad de estar casada es un 31\% inferior. Entre los hombres se da el efecto contrario, el hombre con discapacidad se va acercando con la edad a la probabilidad de casarse que tiene el hombre sin discapacidades. Se pasa de una ratio de 0,62 en el grupo 20 a 39 años, hasta una de 0,91 en los hombres de 60 y más años, esto es, los hombres mayores con discapacidades tienen una probabilidad de estar casados un $9 \%$ menor que los hombres sin discapacidades0. Estos datos ilustran cómo el acceso a los recursos afectivos puede o podría estar mediado (al menos) por tres variables diferentes: género, edad y discapacidad.

Teniendo en mente esta interseccionalidad, estas variables transitan la matriz de dominación que explica cómo interactúan diferentes sistemas de poder (Collins, 2000: 227-228 en Collins, 2017): capacitismo, heteropatriarcado, capitalismo...De manera sucinta, desde el enfoque interseccional y aun a riesgo de simplificar en exceso: no es lo mismo ser mujer que hombre en una sociedad patriarcal como la nuestra, del mismo modo que tampoco lo es ser mujer con una diversidad funcional o no serlo, o ser una mujer 
que ha crecido en un entorno socioeconómico superior a la media a hacerlo en una familia de clase baja.

Estas desigualdades - a veces independientes, a veces cruzadas - deben tenerse en cuenta a la hora de analizar las diferentes dificultades de las personas con diversidad funcional en el alcance y consecución de un proyecto de vida independiente y autónoma, y cómo no, a la hora de analizar su acceso al capital afectivo-sexual.

\section{Interseccionalidad política}

Existen varias clasificaciones para encuadrar la distintas políticas públicas en un eje de interseccionalidad/no interseccionalidad. En primer lugar y en el punto más alejado de la perspectiva interseccional encontramos el "enfoque unitario" acuñado por Hancock (2007) (también denominado single issue). Este describe una perspectiva dirigida a un problema concreto (desigualdad de género, diversidad funcional, orientación sexual...) e inevitablemente, deja de alumbrar las diferentes necesidades de las personas como fruto de la heterogeneidad de las identidades individuales. En un segundo nivel, el modelo múltiple trataría varias desigualdades de manera independiente. En el último nivel, el modelo interseccional iría un paso más allá de esta visión del modelo múltiple poniendo el acento en la interdependencia entre distintas líneas de opresión y subrayando el posible hecho de que las personas ocupen posiciones diferentes de opresora y oprimida simultáneamente (Coll-Planals y Cruells, 2013) en función de la combinación de estos diversos ejes de desigualdad (género, diversidad funcional, clase, orientación sexual...).

Por tanto, el enfoque interseccional no trata sencillamente de hacer notar que todas las personas están atravesadas por ese cruce de desigualdades que las colocan en un lugar de menor acceso a bienes y recursos, sino que trataría de dilucidar cuáles son esos mecanismos interseccionales de desigualdad que acaban afectando a unas determinadas personas en un determinado contexto y en un determinado momento (en este caso, género, diversidad funcional, clase, etnia...). En este sentido, uno de los aportes esenciales de Crenshaw (1994) supuso el hecho de cuestionar al sujeto mujer blanca, heterosexual y de clase media, desvelando las vivencias de otras mujeres a través de la crítica a las políticas monofocales de género que se estaban llevando a cabo. Como mencionábamos al principio, y a modo de ejemplo, tradicionalmente el movimiento feminista ha luchado por el derecho a la interrupción voluntaria del embarazo, mientras que las demandas de las mujeres con discapacidad parecen diametralmente opuestas y reclaman el derecho a ser madres. Del mismo modo, una parte del feminismo lucha por desfeminizar el ámbito del cuidado mientras que las demandas de las mujeres con diversidad funcional apuntan a la posibilidad de ejercer la crianza y el cuidado de su descendencia. Por consiguiente, la perspectiva interseccional cobra especial relevancia en el ámbito que nos ocupa: los DDSSRR de las mujeres con diversidad funcional, al experimentar desigualdades cruzadas que las posicionan en un determinado estado de desigualdad con respecto a otras mujeres, así como con respecto a los hombres con diversidad funcional así como dentro del mismo grupo de las mujeres con diversidad funcional.
La última Observación general número 3 del Comité de los Derechos de las Personas con Discapacidad de las Naciones Unidas (2016) en su artículo 2 afirma que "se dispone de pruebas sólidas que demuestran que las mujeres y las niñas con discapacidad afrontan obstáculos en la mayor parte de los ámbitos de la vida [...] [que] generan situaciones en las que existen formas múltiples e interseccionales de discriminación". Estas posibles intersecciones pueden incluir motivos de discriminación tales como "la edad; la discapacidad; el origen étnico, indígena, nacional o social; la identidad de género; la opinión política o de otra índole; la raza; la condición de migrante, refugiado o solicitante de asilo; la religión; el sexo y la orientación sexual" (artículo 4).

¿Se presta atención a esta interseccionalidad en el ámbito de los DDSSRR en nuestro país? La realidad es que se observa una falta de perspectiva de género en las políticas sobre discapacidad y viceversa, un déficit de enfoque de diversidad funcional en las de género. En primer lugar, existe una carestía de datos estadísticos oficiales. Por ejemplo, la Encuesta Nacional sobre Salud Sexual y Reproductiva de 2009 no posee una cuestiónexplícita donde se pregunte si la persona entrevistada posee algún tipo de discapacidad. Asimismo los sesgos capacitistas y de género se reproducen en la normativa y son muchos los países que han dictado leyes sobre la prohibición del matrimonio a personas con discapacidad que esconden un sesgo capacitista y eugenésico por el miedo a que estas mujeres tengan hijos e hijas con su misma enfermedad (Ash y Fine, 1992). En nuestro país, el Congreso recientemente aprobó la modificación de la Ley de Jurisdicción Voluntaria del Código Civil, cuyo objetivo es que las personas con discapacidad puedan contraer matrimonio sin necesidad de presentar un dictamen médico (Cermi, 2017).

Por otro lado, la adopción puede resultar imposible para muchas mujeres con discapacidad (Gomiz, 2016; Peláez, 2009) así como la inseminación o ganar la custodia de los hijos e hijas tras el divorcio (Peláez, 2009). En el caso concreto de la adopción resulta complicado obtener el Certificado de Idoneidad (requisito indispensable para la adopción), especialmente cuando afecta a la mujer, por ser ella la víctima mayoritaria en los casos de rechazo. Este Certificado requiere "disfrutar de un estado de salud, física y psíquica, que no dificulte el normal cuidado del menor" (Peláez, 2009). La ambigüedad de este apartado deja la puerta abierta a la discrecionalidad.

Asimismo, la Ley 39/2006, de 14 de diciembre, de Promoción de la Autonomía Personal y Atención a las personas en situación de dependencia reproduce a lo largo de todo su contenido el mito de la persona con diversidad funcional como cuidada y no cuidadora. En la puesta en práctica de la misma con respecto a la asistencia personal se ha acabado enfocando este servicio al trabajo reproductivo perpetuando así sesgos de género y capacitistas.

iDIVERSITAR LA SEXUALIDAD! iDIVERSITAR LA MATERNIDAD! iDIVERSITAR LA VIDA!

Merece la pena recordar el siempre actual lema feminista que nos dejó Kate Millet de "lo personal es político". Bajo esta premisa, el sexo es político. La maternidad es política. 
Anne Finger (1992), referente en el activismo de los derechos de las personas con diversidad funcional afirmó que para ellas la sexualidad era la fuente de su más profunda opresión y a menudo la fuente de su más profundo dolor. La historia de los derechos de las personas con diversidad funcional ha estado marcada por un discurso ausente del placer que ha perpetuado su estatus de víctimas y de personas asexuales. Por tanto,se debe tener en mente que la plena accesibilidad pasa necesariamente por el acceso al placer (Tepper, 2000). Así pues -como llamaba la atención la CDPD en 2006- los derechos sexuales y reproductivos, incluyendo el respeto del hogar y de la familia (artículos 23 y 25), continúan siendo diez años después uno de los aspectos de los Derechos Humanos de las personas con discapacidad menos atendidos y vulnerados de manera sistemática (en especial, como sabemos, en el caso de las mujeres y niñas).

Las palabras de Kate Millet y de Anne Finger nos recuerdan que las luchas del feminismo y de la diversidad funcional tienen mucho en común y ambas deberían ir encaminadas a eliminar las leyes, normas, prácticas y estructuras que releguen a las personas a una característica biológica (Ash y Fine, 1992) para así huir del relato biomédico también en el ámbito sexual y reproductivo. Nuestros cuerpos (todos) no se encasillan en enfermos o sanos, nuestras prácticas sexuales no pasan necesariamente por los genitales y no solo somos madres por el hecho de parir.

La realidad es que resulta difícil encajar en el ideal de sujeto reproductivo y sujeto sexual para todas las personas o al menos hacerlo en todo momento. ¿Por qué no tumbar este orden que establece un centro y unos márgenes en la sexualidad y la reproducción? ¿No pasaría una sexualidad más enriquecedora por "poner la diversidad como norma y no como excepción"? (Ministerio de Sanidad, Política Social e Igualdad, 2011: 31). Como detalla Wilkerson (2002) existe una erotofobia cultural hacia las personas con diversidad funcional y debe ser derribada. ¿Qué pasaría si la diversidad funcional fuera sexy? ¿Y si las personas con diversidad funcional fueran concebidas como objetos y sujetos de deseo de una multiplicidad de deseos y prácticas? (McRuer y Mollow, 2012). Diversitar el sexo (disabling sex) surge como una oportunidad no solo para las personas con diversidad funcional sino para todas las personas (Shakespeare, 1999). Es decir, explorar una sexualidad diversa que nos haga escapar del yugo del coitocentrismo, del falocentrismo y de la imagen de los encuentros sexuales como un espectáculo de equilibrismos varios.

El sujeto reproductivo que ostenta el privilegio es, como hemos visto, una mujer sin discapacidad, heterosexual y con el género que se le ha asignado al nacer (Kalender, 2010). Las mujeres con diversidad funcional se encuentran en los márgenes de la institución de la maternidad normativa, pero sin embargo, basándonos en su experiencia, la realidad es que negocian con las tensiones del ideal de maternidad, algo que permite ver los retos de esta construcción para todas las madres (Malacrida, 2009). Repetimos: para todas las madres. ¿Por qué no diversitar entonces la maternidad? Diversitar la maternidad cobra sentido en un contexto de interdependencia en el que todas las personas necesitamos apoyos, recursos y servicios para realizar la ardua tarea de la crianza, máxime en el momento actual de retroceso del Estado de Bienestar. ¿No son estas demandas propias de todas las personas?

Por tanto, la lucha es la misma. En un entorno mediado por tal multitud de circunstancias que se torna más y más complejo con el devenir de los años, ¿quién encaja en el estricto troquel de la "norma"? Asumir la diversidad como un continuo del género y viceversa que no es más que un desafío al imperio del cuerpo del hombre, blanco, heterosexual, de clase media...Teniendo en cuenta el punto de vista interseccional, la realidad es que todos y todas en algún punto somos disidentes de este sistema heteronormativo, capacitista, capitalista, racista, clasista... Por esta razón, se hace necesario reclamar la apertura del sujeto sexual y reproductivo (Kalender, 2010) como demanda central del feminismo que queremos. Parafraseando a Rubin, ha llegado el momento de pensar sobre la diversidad. Ha llegado el momento de diversitar la vida.

\section{REFERENCIAS}

Arnau, Soledad. 2005. Otras voces de mujer: El feminismo de la diversidad funcional. Asparkía: Investigació Feminista, 16.

Ash, Adrienne y Fine, Michelle. 1992. Beyond Pedestals: Revisiting the Lives of Women with Disabilities. En Fine, Michelle, Critical Perspectives on Women and Gender. Disruptive Voices: The Possibilities of Feminist Research, 139-171. Ann Arbor: University of Michigan Press.

Bonnie, Selina. 2004. Disabled People, Disability and Sexuality. En John Swain, Disabling barriers-enabling environments (2nd ed.), 125-133. London: SAGE Publications.

Casado, Elena. 1998. A vueltas con el sujeto del feminismo. Revista Internacional de Sociología, Tercera Época, 21: 73-91.

CERMI, 2017. Las personas con discapacidad podrán casarse sin presentar dictamen médico. Cermi, 22/07/2017.

Coll-Planas, Gerard y Cruells, Marta. 2013. La puesta en práctica de la interseccionalidad política: el caso de las políticas LGTB en Cataluña. Revista Española de Ciencia Política, 31: 153-172.

Collins, Patricia. 2017. The Difference That Power Makes: Intersectionality and Participatory Democracy. Revista de Investigaciones Feministas, 8(1): 19-39.

Comité de los Derechos de las Personas con Discapacidad de las Naciones Unidas. 2016. Observación general número 3.

Convención sobre los Derechos de las Personas con Discapacidad de las Naciones Unidas. 13 de diciembre de 2006. Consultado el 23/05/2017. http://www.un.org/ esa/socdev/enable/documents/tccconvs.pdf

Crenshaw, Kimberlé. 1994. Mapping the Margins: Intersectionality, Identity Politics, and Violence Against Women of Color. En Fineman, Martha y Mykitiuk, Roxane (eds.) The Public Nature of Private Violence, 93-108. New York: Routledge.

Cruz, Pilar. 2004. Mujeres con discapacidad y su derecho a la sexualidad. Politica Y Cultura, 22: 147-160.

EFE. 2005. Un desnudo diferente en el centro de Londres. EI País, 15/09/2005.

Finger, Anne. 1992. Forbidden Fruit. New Internationalist, 233.

Fundación CERMI Mujeres, Foro Europeo de la Discapacidad. 
2017. Poner fin a la esterilización forzosa de las mujeres y niñas con discapacidad. Madrid: CERMI Mujeres y EDF.

Fundación CERMI Mujeres. 2018. Aportaciones de la Fundación CERMI Mujeres al borrador de Observación General preparado por el Comité sobre los Derechos de las Personas con Discapacidad sobre los artículos 4.3 y 33.3 en materia de implementación y monitoreo de la CRPD. Madrid: Fundación CERMI Mujeres.

Gomiz, María del Pilar. 2016. La sexualidad y la maternidad como factores adicionales de discriminación (y violencia) en las mujeres con discapacidad. Revista Española De Discapacidad (REDIS), 4(2): 123-142.

Hancock, Ange-Marie. 2007. When Multiplication Doesn't Equal Quick Addition: Examining Intersectionality as a Research Paradigm. Perspectives on Politics, 5(1): 63-79.

Instituto Nacional de Estadística. 2008. Encuesta sobre Discapacidades, Deficiencias y Estado de Salud.

Kalender, Ute. 2010. Nothing beyond the able mother? A queer-crip perspective on notions of the reproductive subject. German feminist bioethics. International Journal of Feminist Approaches to Bioethics, 3(2): 150-169.

Kent, Deborah. 1987. Disabled women: portraits in fiction and drama. En Alan, Gartner y Tom, Joe (eds.) Images of Disabled. Disabling Images, 47-63. London: Praege.

Ley 39/2006, de 14 de diciembre, de Promoción de la Autonomía Personal y Atención a las personas en situación de dependencia. https://www.boe.es/buscar/ act.php?id=BOE-A-2006-21990

López, María. 2008. Mujeres Con Discapacidad: Mitos y realidades en las relaciones de pareja y en la maternidad. Madrid: Narcea Ediciones.

Malacrida, Claudia. 2009. Performing motherhood in a disablist world: dilemmas of motherhood, femininity and disability. International Journal of Qualitative Studies in Education, 22(1): 99-117.

McRuer, Robert y Mollow, Ana. 2012. Sex and disability. Duke University Press.

Ministerio de Sanidad, Política Social e Igualdad. 2011. Estrategia Nacional de Salud Sexual y Reproductiva. Madrid: Ministerio de Sanidad, Política Social e Igualdad.

Morris, Jenny. 1997. Encuentros con desconocidas Feminismo y discapacidad (Mujeres). Madrid: Narcea.

Morris, Jenny. 2008. Lo personal y lo político. Una perspectiva feminista sobre la investigación de la discapacidad física. En Len Barton, Superar las barreras de la discapacidad. 18 años de Disability and Society (Sociología Manuales), 315-326. Madrid: Morata.

Organización Mundial de la Salud. 2003. Salud Reproductiva. El Proyecto de estrategia para acelerar el avance hacia el logro de los objetivos y metas internacionales de desarrollo. Ginebra: Organización Mundial de la Salud.

Organización Mundial de la Salud, 2009. Promoting sexual and reproductive health for persons with disabilities WHO/UNFPA guidance note. Ginebra: Organización Mundial de la Salud.

O'Toole, Corbet Joan. 2002. Sex, Disability and Motherhood: Access to Sexuality for Disabled Mothers. Disability Studies Quarterly, 22(4): 81-101.

Peláez, Ana. 2009. Maternidad y discapacidad. Colección Barclays Igualdad y Diversidad, 1.

Preciado, Beatriz. 2011. Genealogía somatopolítica y marcos de inteligibilidad corporal: del cuerpo soberano al cuerpo biopolítico. Seminario Cuerpo Impropio. Guía de modelos somatopolíticos y de sus posibles usos desviados, 2 4/11/2011. Sevilla.

Público. 2012. Los discapacitados piden a Gallardón que elimine la esterilización forzosa. Público, 27/07/2012.

Rich, Adrienne. 1996. Nacemos de mujer la maternidad como experiencia e institución. Feminismos Clásicos. Madrid: Cátedra Instituto De La Mujer.

Rubin, Gayle. 1986. El tráfico de mujeres: Notas sobre la "economía política" del sexo. Revista Nueva Antropología, VIII(30): 95-145.

Shakespeare, Tom. 1999. The Sexual Politics of Disabled Masculinity. Sexuality and Disability, 17(1): 53-64.

Sheldon, Alison. 2004 Women and Disability. En Swain, John, Disabling barriers-enabling environments (2nd ed.), 125133. London: SAGE Publications.

Tepper, Mitchell. 2000. Sexuality and Disability: The Missing Discourse of Pleasure. Sexuality and Disability, 18(4): 283290.

Trujillo, Gracia. 2009. Del sujeto político la Mujer a la agencia de las (otras) mujeres: el impacto de la crítica queer en el feminismo del Estado español. Política y Sociedad, 46(1 y 2): 161-172.

Walsh-Gallagher, Dimpna; Sinclair, Marlene y Mc Conkey, Roy. 2012. The ambiguity of disabled women's experiences of pregnancy, childbirth and motherhood: A phenomenological understanding. Midwifery, 28(2): 156162.

Wates, Michele. 2004. Righting the Picture: Disability and Family Life. En Swain, John, Disabling barriers-enabling environments (2nd ed.), 135-142. London: SAGE Publications.

Wilkerson, Abby. 2002. Disability, Sex Radicalism, and Political Agency. NWSA Journal, 14(3): 33-57. 